\title{
Synergistic Control of Plug-In Vehicle Charging and Wind Power Scheduling
}

\author{
Chiao-Ting Li, Member, IEEE, Changsun Ahn, Huei Peng, and Jing Sun, Fellow, IEEE
}

\begin{abstract}
Significant synergy exists between plug-in electric vehicles (PEVs) and wind energy: PEVs can be the demand response to mitigate the intermittent wind power outputs, and wind energy can provide low-carbon electricity to PEVs. This paper presents a hierarchical control algorithm to realize this synergy by integrating the PEV charging and wind power scheduling. The control algorithm consists of three levels: the top-level controller optimizes the scheduling for the conventional power plants and wind power; the middle-level controller plans PEV charging to achieve load following based on the battery state of charge and plug-off time of each vehicle; the bottom-level controller uses grid electricity frequency as the feedback cue to control PEV charging and serves as the ancillary service to the grid. Numerical simulations show that the integrated controller can improve the grid frequency regulation and overall electricity generation cost without sacrificing the PEVs charging completion.
\end{abstract}

Index Terms-Charging of electric vehicles, electricity grid, grid integration, plug-in electric vehicles, smart grid, wind energy.

\section{INTRODUCTION}

$\mathbf{P}$ LUG-IN electric vehicles (PEVs) and wind energy are both green technologies capable of reducing the use of fossil fuels, and, due to the aggressive goals to reduce greenhouse gas emissions and carbon footprints around the world, they are expected to grow quickly in the next decades. However, accommodating large number of PEVs and wind energy sources poses challenges to the electricity grid because of the increased load from PEV charging and intermittency from wind power. Fortunately, there is significant synergy between PEVs and wind power [1], [2], but few studies to take advantage of this synergy were reported. This paper intends to bridge the gap by proposing a control algorithm to integrate PEV charging and wind power scheduling.

Charging a large number of PEVs without mitigation will negatively impact the electricity grid [3], [4]. However, the deployment of advanced metering and charging systems makes it possible to control the timing and rate of PEV charging. In addition, PEVs often have the dwell time (stay parked) longer than

Manuscript received January 05, 2012; revised January 30, 2012, April 02, 2012, June 07, 2012, and July 20, 2012; accepted July 29, 2012. Date of publication September 11, 2012; date of current version April 18, 2013. This work was supported by the National Science Foundation under Grant No. 0835995. Paper no. TPWRS-00014-2012.

C.-T. Li, C. Ahn, and H. Peng are with the Department of Mechanical Engineering, University of Michigan, Ann Arbor, MI 48109 USA (e-mail: ctli@umich.edu; sunahn@umich.edu; hpeng@umich.edu).

J. Sun is with the Department of Naval Architecture and Marine Engineering, University of Michigan, Ann Arbor, MI 48109 USA (e-mail: jingsun@umich. edu).

Color versions of one or more of the figures in this paper are available online at http://ieeexplore.ieee.org.

Digital Object Identifier 10.1109/TPWRS.2012.2211900 the time needed to fully charge their batteries, which provides control algorithms some degree of freedom to delay or manipulate the charging.

Controlling PEV charging is more challenging than managing existing grid load in that both the charging timing (service timing) and charging rate (service quantity) are control variables, which current demand-side management or service provisioning schemes rarely address simultaneously. For example, resource allocation methods [5]-[7] only address the service quantity, whereas scheduling [8] and queuing theory [9]-[11] only address the timing. In addition, these methods are highly centralized, which limits themselves to be used on large PEV fleets. Hierarchical and partially-decentralized algorithms are believed to be more appropriate for PEV charging [12]. For schemes specific to PEV charging, the dual tariff (cheaper electricity rate in the valley hours) is currently offered by several electricity distribution companies [13], [14]. Rule-based charging schemes are used to analyze PEVs' impact on the grid generation mix and carbon emissions [3], [15]. Cost optimization has been able to achieve valley filling [16]. Co-optimization for costs of electricity and heating service [17] and using idle PEVs as grid reserves [18] have also been proposed. However, rule-based approaches do not exploit the full potential of the controllable PEV charging whereas cost optimizations are usually solved off-line without providing implementable real-time control laws, with [19] being a notable exception.

Wind power is cleaner than most traditional power sources, but its intermittency has been a concern to grid reliability. Although aggregating outputs of multiple turbines or wind farms help to smooth the fluctuations [20], the grid operator may still need to procure more reserves for the wind intermittency [21], which can cost \$0.45-\$8.84/MWh [22].

Researchers are tackling the wind intermittency in various ways. Some research efforts have focused on reducing prediction error of wind power to assist grid operations [23]. Another research direction focuses on developing strategies for scheduling generation sources. Several studies included wind energy probabilities when conducting optimizations for generation scheduling [24]-[27]. Bidding strategy to maximize the profit for the wind energy producer (not the whole grid) was also proposed [28]. Fuzzy logic has been used as a scheduling scheme as well [29]. Despite of the improvement achieved by the above methods, curtailment of wind power will still happen because the current electricity grid has very little energy storage capacity.

The literature has addressed the PEV charging or wind intermittency on the electricity grid. Their integration is attracting 

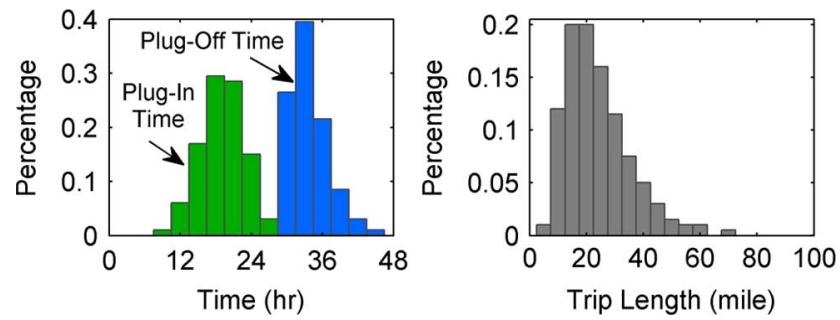

Fig. 1. Distributions of the plug-in time, plug-off time, and trip length [31]. The distribution of trip length is used to derive $S O C_{\mathrm{ini}}$.

increasing attention because of the significant synergy between them. The PEV charging power can be throttled back and forth to cancel the intermittency in wind energy, and wind energy can provide clean electricity to PEVs. This paper proposes a threelevel hierarchical control algorithm to achieve such integration. The top-level controller optimizes the scheduling for the conventional power plants and wind power to minimize the electricity generation cost; the middle-level controller plans PEV charging to achieve load following based on the battery state of charge and plug-off time of each vehicle; the bottom-level controller uses grid electricity frequency as the feedback cue to control PEV charging and serves as the ancillary service to the grid. This control algorithm is an extension of the algorithms in [19] and [30], which can fully exploit the synergy between PEV and wind energy to achieve multiple objectives on the electricity grid.

The remainder of this paper is organized as follows: Section II describes models of the PEV fleet, wind power, and the electricity grid; Section III presents the three-level hierarchical control algorithm; Section IV shows simulation results of the controller on a state-wide electricity grid; and Section V provides concluding remarks.

\section{MODELING}

Several models are developed to describe system-level dynamics of the PEV fleet, wind power, and electricity grid using statistics in the State of Michigan. In addition, PEVs and wind energy are assumed to have acquired sizable market shares, so they have significant influence on the demand, supply, and reliability of the electricity grid.

\section{A. Plug-In Electric Vehicle Fleet}

The total number of PEVs is assumed to be two million, which corresponds to $25 \%$ of the vehicle fleet in Michigan. All PEVs are assumed to use smart chargers and thus controllable. The PEV fleet is characterized by the plug-in time, the plug-off time, and the state of charge (SOC) of the battery at plug-in. The SOC quantifies the energy requirement to fully charge all PEVs so that the grid operator can schedule appropriate power generation to accommodate the PEV charging need, and the plug-in/plug-off time prioritizes the PEV fleet and determines which vehicle receives immediate or delayed charging service. The data in [31], shown in Fig. 1, is used to derive the three pieces of information.

Assuming that charging only happens at home, the arrival time is treated as the plug-in time, and the departure time as the plug-off time. The trip length is used to derive the SOC

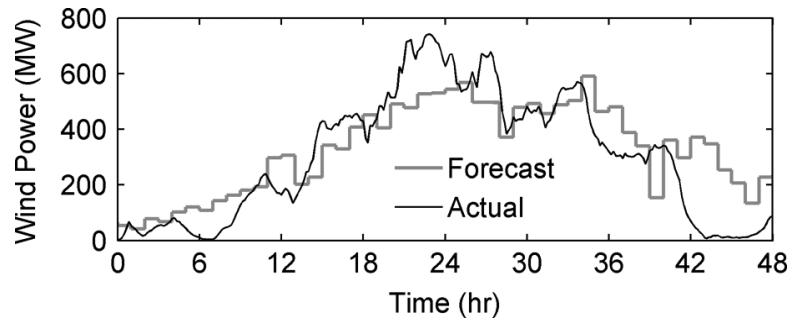

Fig. 2. A 48-h snapshot of the NREL eastern wind dataset. The raw data consists of two data strings: wind forecast, $w_{\mathrm{f}}$, and actual wind generation, $w_{\mathrm{a}}$.

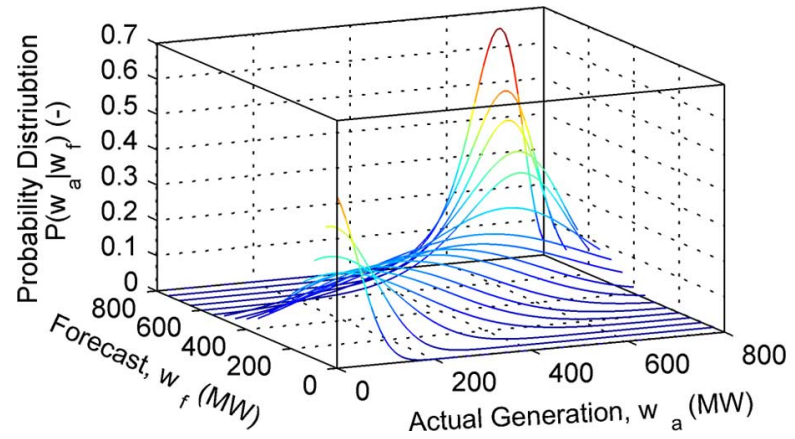

Fig. 3. Conditional probability distributions, $\mathbb{P}\left(w_{\mathrm{a}} \mid w_{\mathrm{f}}\right)$. The peak value of each distribution is close to the forecast value, $w_{\mathrm{f}}$, implying that the forecast is generally good.

at plug-in by (1), which then is used to find the total energy requirement to charge the PEV fleet using (2):

$$
\begin{aligned}
S O C_{\mathrm{ini}} & = \begin{cases}0.8-(0.8-0.3) \times \frac{L}{\mathrm{AER}}, & \text { if } L<\mathrm{AER} \\
0.3, & \text { otherwise }\end{cases} \\
K & =\sum_{i=1}^{N}\left(0.8-S O C_{\mathrm{ini}}\right)_{i} \times Q
\end{aligned}
$$

where $L$ is the trip length and AER is the all-electric range of the PEV. The initial SOC will lie in the window of $30-80 \%$, which is the range the battery operates [32]. $Q$ is the battery capacity and is assumed to be $16 \mathrm{kWh}$, which is typical for 40 -mile-AER PEVs [32]. The maximum charging power is $1440 \mathrm{~W}$, limited by the Level-I electric vehicle charger [33], and only night charging at home is allowed. The response of the charging level is assumed to be instantaneously fast, and dynamics and losses in batteries are ignored.

\section{B. Wind Power}

An $800-\mathrm{MW}$ wind farm is assumed to be connected to the electricity grid, which can support about $10 \%$ of the peak load in Michigan when running at the rated power. The Eastern Wind Dataset from the National Renewable Energy Laboratory (NREL) [34] is used to extract the conditional probability distribution, denoted as $\mathbb{P}\left(w_{\mathrm{a}} \mid w_{\mathrm{f}}\right)$, to represent the (stochastic) actual wind generation $\left(w_{\mathrm{a}}\right)$ under a given forecast $\left(w_{\mathrm{f}}\right)$. Fig. 2 shows a snapshot of the NREL dataset, and Fig. 3 shows the extracted conditional probability distribution. $\mathbb{P}\left(w_{\mathrm{a}} \mid w_{\mathrm{f}}\right)$ will be used to derive the reserve requirement and to estimate the expected shortfall of wind power.

The grid operator procures reserve to handle the wind intermittency and other uncertainties. Assuming that over-production in wind power can always be curtailed, only the up-regu- 


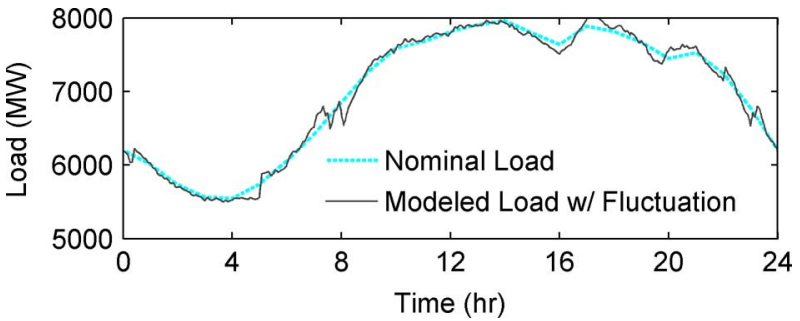

Fig. 4. Nominal and modeled load profiles.

lation reserve is needed for under-production. The curtailment allows wind power producers to reduce risks in the electricity market, in that they do not need to pay for over-production. Furthermore, the wind power producers can schedule their generation conservatively (lower than forecasts) when the energy price in the market is low or when the reserve price is high. The wind scheduling can be aggressive when they are not fully responsible for their intermittency (which is the case in many markets today). A further assumption is that the reserve has to cover $95 \%$ of the under-production, and then the reserve requirement, $R_{\mathrm{w}, \mathrm{rqd}}$, can be computed using (3). The expected shortfall, $w_{\text {short }}$, shown in (4), must be made up by dispatching the reserve or throttling back the PEV charging:

$$
\begin{aligned}
R_{\mathrm{w}, \mathrm{rqd}} & =\left[w_{\mathrm{s}}-\mathbb{F}^{-1}(0.05)\right]^{+} \\
w_{\text {short }} & =\mathbf{E}\left\{\left[w_{\mathrm{s}}-w_{\mathrm{a}}\right]^{+}\right\}
\end{aligned}
$$

where the two aforementioned quantities are both functions of the wind scheduling decision, $w_{\mathrm{s}}$, which is a control variable in the scheduling optimization in Section III-A. $\mathbb{F}$ is the cumulative probability distribution function of $\mathbb{P}\left(w_{\mathrm{a}} \mid w_{\mathrm{f}}\right)$, and $\mathbb{F}^{-1}$ is the inverse of $\mathbb{F}$, which tells the least wind power output of a given probability. The plus sign $(+)$ indicates the truncation of negative values, and the expectation (the operation imposed by E) in (4) is taken with respect to $w_{\mathrm{a}}$.

\section{Electricity Grid}

The grid model consists of several elements: the load, generation costs, and frequency dynamics.

1) Grid Load: The hourly load data in the Detroit Edison serviced area is used as the nominal load, which ranges between 5500-8000 MW. Sub-hour fluctuations are generated by a random process to match the typical range of fluctuations on state-wide power systems [35]. The nominal load and modeled load with fluctuations are shown in Fig. 4.

On top of the existing grid load, $7.38 \mathrm{GWh}$ of energy is required to charge the two million PEVs. The PEV charging is assumed to be controllable, and the optimal charging is to be found by the optimization in Section III-A.

2) Generation Costs: The price of electricity generation, shown in Fig. 5, is acquired from the Oak Ridge Competitive Electricity Dispatch Model [36]. The reserve scheduling cost is assumed to be $3 \%$ more expensive than the generation price based on the statistics in [37], and the reserve dispatch cost is assumed to be the same as the generation price and only occurs if the reserve is dispatched.

3) Grid Frequency Dynamics: The frequency of the AC electricity deviates from the nominal $60 \mathrm{~Hz}$ when there is mismatch

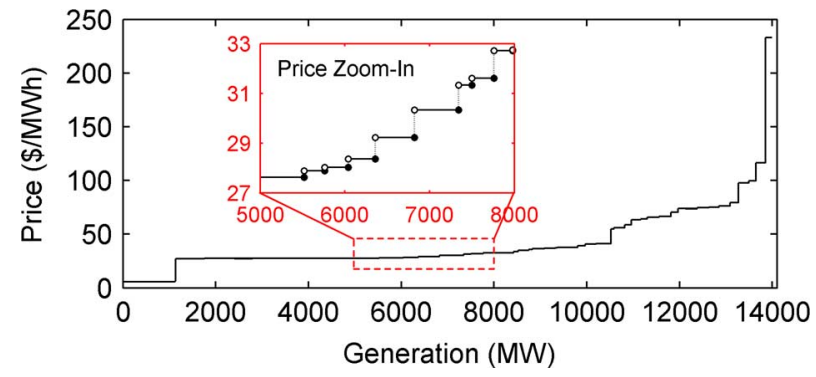

Fig. 5. Price of electricity generation. This curve increases in a staircase fashion because the dispatch of power plants is discrete and the price jumps once an additional power plant is switched on.

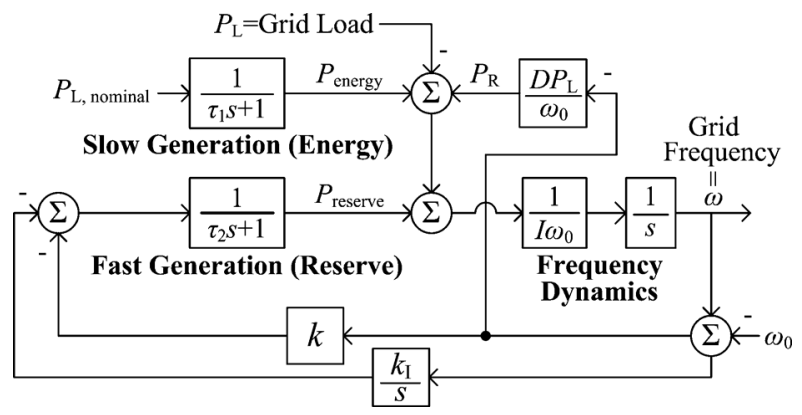

Fig. 6. Model of the grid frequency dynamics.

between the load and generation. The grid frequency dynamics is approximated by the rotational dynamics shown in (5), and its model structure is depicted in Fig. 6:

$$
\dot{\omega}=\frac{P_{\text {surplus }}}{I \omega_{0}}=\frac{-P_{\mathrm{L}}+P_{\text {energy }}+P_{\text {reserve }}+P_{\mathrm{R}}}{I \omega_{0}}
$$

where $I$ is the system inertia, and $\omega_{0}$ is the nominal frequency. $P_{\mathrm{L}}$ is the grid load, $P_{\text {energy }}$ and $P_{\text {reserve }}$ are the electricity generation and reserve. The last term, $P_{\mathrm{R}}$, is frequency dependent to capture the phenomenon that rotary loads, such as motors, slow down and consume less electricity when the grid frequency decreases, which is modeled as (6):

$$
P_{\mathrm{R}}=D P_{\mathrm{L}}\left(\frac{\omega_{0}-\omega}{\omega_{0}}\right)
$$

where $D$ is an empirical constant suggested in [38].

Equation (7) and (8) describe the dynamics of $P_{\text {energy }}$ and $P_{\text {reserve }}$. Both are first-order dynamics, but with different time constants and implications:

$$
\begin{aligned}
& \dot{P}_{\text {energy }}=-\frac{1}{\tau_{1}}\left(P_{\text {energy }}-P_{\mathrm{L}, \text { nominal }}\right) \\
& \dot{P}_{\text {reserve }}=-\frac{1}{\tau_{2}}\left\{P_{\text {reserve }}-\left[-k\left(\omega-\omega_{0}\right)-k_{I} \int\left(\omega-\omega_{0}\right) d t\right]\right\}
\end{aligned}
$$

where $\tau_{1}$ and $\tau_{2}$ are time constants, $P_{\mathrm{L}, \text { nominal }}$ is the nominal grid load, $k$ and $k_{\mathrm{I}}$ are controller gains. $\tau_{1}$ and $\tau_{2}$ are chosen to resemble the ramp rate limits in each type of generation [38], and $k$ and $k_{\mathrm{I}}$, are chosen to match typical frequency deviations on state-wide grids [39]. 


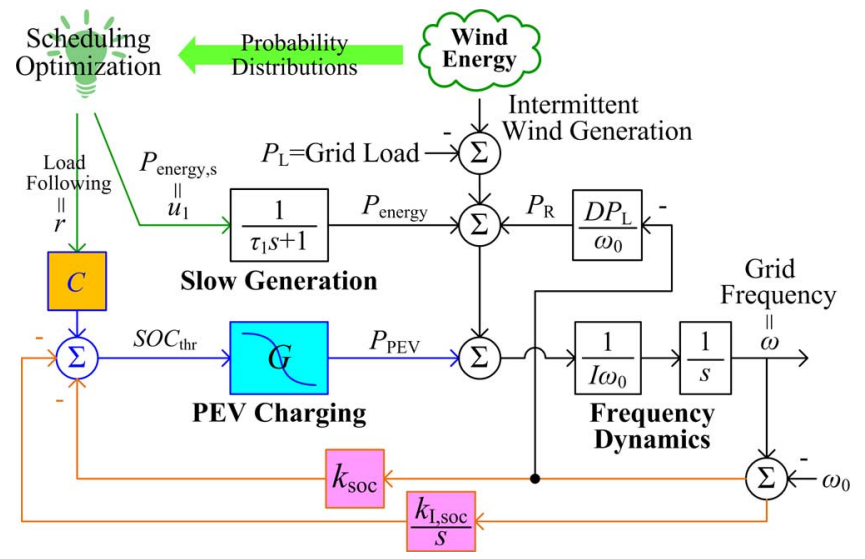

Fig. 7. New model of the grid frequency dynamics with controllers for wind power scheduling and PEV charging included.

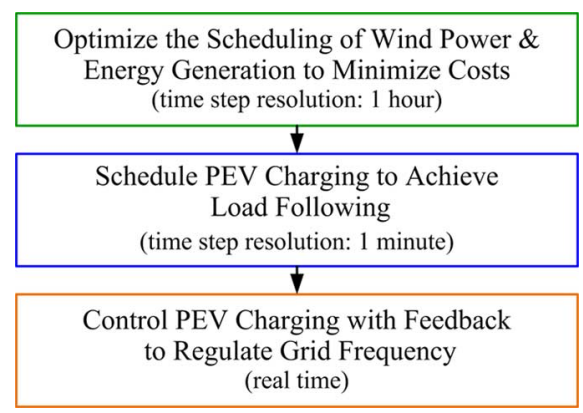

Fig. 8. Objectives and time scales of the hierarchical controller.

\section{HierarchicAl Controller}

A hierarchical controller is designed to incorporate the PEV charging and wind power into the electricity grid. The grid structure is changed from Figs. 6 and 7. The controller consists of three levels, and wind power stochastic is included into the toplevel controller (marked as the green light bulb), and the PEV charging is handled by the middle-level $(C$ and $G$ ) and bottomlevel controllers $\left(k_{\mathrm{soc}}\right.$ and $\left.k_{\mathrm{I}, \mathrm{soc}}\right)$. The objectives and time step resolutions of each controller are summarized in Fig. 8.

\section{A. Top-Level Controller: Scheduling Optimization}

An optimization problem is formulated to find the optimal scheduling of the electricity generation and wind power to satisfy the grid load and PEV charging demand at minimum cost. The optimization problem is stated in (9)-(19). Equation (9) is the objective function, including costs of electricity generation $\left(C_{\text {energy }}\right)$, reserve scheduling $\left(C_{\text {reserve,s }}\right)$, and expected reserve dispatch $\left(C_{\text {reserve,d }}\right)$. The rewards to PEVs for supporting reserves, if considered, can also be included into the objective function; however, such remuneration is not considered in this work. The two controlling variables, $u_{1}$ and $u_{2}$, are the electricity generation $\left(P_{\text {energy }, \mathrm{s}}\right)$ and the scheduling of wind power $\left(w_{\mathrm{s}}\right)$. The state, $x$, is the remaining PEV energy demand. Its dynamics and constraints are detailed as follows:

$$
\min _{u_{1}, u_{2}} \sum_{t=1}^{T}\left\{C_{\text {energy }}\left(u_{1}\right)+C_{\text {reserve }, \mathrm{s}}\left(R_{\mathrm{s}}\right)+C_{\text {reserve, } \mathrm{d}}\left(R_{\mathrm{d}}\right)\right\}
$$

subject to

$$
\begin{aligned}
& u_{1}(t)+u_{2}(t)-P_{\mathrm{L}, \text { nominal }}(t)=P_{\mathrm{PEV}}(t), \forall t \\
& \sum_{t=1}^{T} P_{\mathrm{PEV}}(t) \cdot \Delta t=K \\
& 0 \leq P_{\mathrm{PEV}}(t) \leq \min \left\{x(t), U_{\mathrm{PEV}}\right\}, \forall t \\
& x(t+1)=x(t)-P_{\mathrm{PEV}}(t) \cdot \Delta t, \forall t \\
& x(0)=K \\
& R_{\mathrm{L}, \mathrm{rqd}}(t)=0.05 \times P_{\mathrm{L}, \text { nominal }}(t), \forall t \\
& R_{\mathrm{w}, \mathrm{rqd}}(t)=\left[u_{2}(t)-\mathbb{F}^{-1}(0.005)\right]^{+}, \forall t \\
& R_{\mathrm{s}}(t)+P_{\mathrm{PEV}}(t) \geq R_{\mathrm{L}, \mathrm{rqd}}(t)+R_{\mathrm{w}, \mathrm{rqd}}(t), \forall t \\
& w_{\text {short }}(t)=\mathbf{E}\left\{\left[u_{2}(t)-w_{\mathrm{a}}\right]^{+}\right\}, \forall t \\
& R_{\mathrm{d}}(t)=\left[w_{\text {short }}(t)-P_{\mathrm{PEV}}(t)\right]^{+}, \forall t
\end{aligned}
$$

where

$P_{\mathrm{PEV}} \quad$ aggregated PEV charging load (MW);

$U_{\mathrm{PEV}} \quad$ charging limit (MW);

$K \quad$ total PEV energy demand (MWh);

$R_{\mathrm{L}, \mathrm{rdq}} \quad$ reserve requirement for grid load (MW);

$R_{\mathrm{W}, \mathrm{rdq}} \quad$ reserve requirement for wind power (MW);

$R_{\mathrm{s}} \quad$ scheduling of conventional reserve (MW);

$W_{\text {short }} \quad$ expected shortfall of wind power (MW);

$R_{\mathrm{d}} \quad$ expected dispatch of conventional reserve (MW).

Equations (10)-(14) are constraints related to the electricity generation: (10) states the balance of supply and demand (i.e., scheduled generation and loads); (11) ensures that the total PEV charging demand is satisfied; (12) states the lower and upper bound on PEV charging power due to the Level-I charger limit; (13) describes the state dynamics; and (14) is the constraint for the initial state.

Equations (15)-(19) are related to the reserve scheduling and dispatch: (15) states the reserve requirement for the grid load, which is $5 \%$ of the nominal load magnitude according to [21]; (16) states the reserve requirement for wind power according to (3); (17) states that the total reserve requirement must be met by either the controllable PEV load or the scheduling of conventional reserve; (18) states the expected shortfall of wind power; and (19) states the expected dispatch of conventional reserve if wind shortfall exceeds the magnitude of the controllable PEV load. Notice that (17) counts the PEV load as reserve because it can be throttled back and forth by the bottom-level controllers (to be explained in Section III-C). Finally, (19) implies that throttling back PEV load is preferred to dispatching the conventional reserve because the former is free.

In addition, several implicit influences of $u_{2}$ in the optimization problem are worth mentioning. Increasing $u_{2}$ can reduce the energy generation $\left[u_{1}\right.$ in (10)], but it also increases the reserve required for wind power $\left[R_{\mathrm{w}, \mathrm{rqd}}\right.$ in (16)] and the expected shortfall [ $w_{\text {short }}$ in (18)]. Consequently, the scheduling and expected dispatch of conventional reserve may rise $\left[R_{\mathrm{s}}\right.$ in (17) and $R_{\mathrm{d}}$ in (19)] if they exceed the range that the controllable PEV load can cover. These coupling constraints are the main reasons why this optimization problem is non-intuitive. 


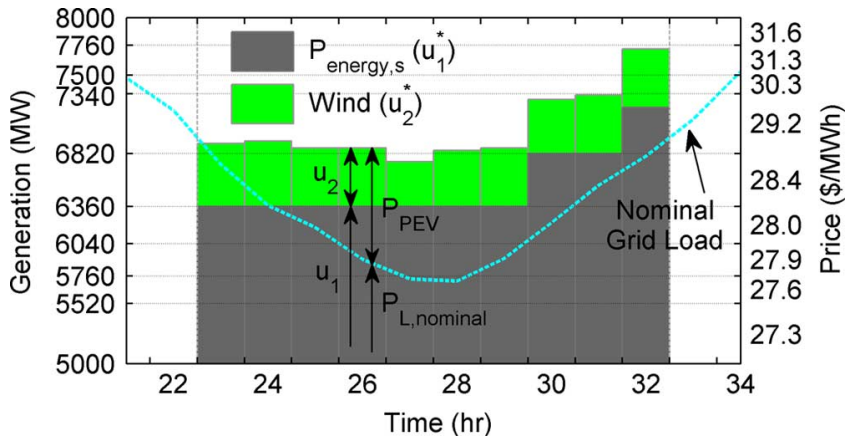

Fig. 9. Optimal scheduling results for the nominal case.

The scheduling optimization is solved assuming that knowledge about generation prices (Fig. 5), the nominal grid load (the dash line in Fig. 4), and wind forecasts (the grey line in Fig. 2) are known. However, the actual grid load and actual wind output are not known. The time horizon of the optimization is $11 \mathrm{PM}$ to $8 \mathrm{AM}$, which are denoted as Hours $23-32$ in several following figures. This time horizon is chosen because we focus on night charging. A longer horizon is unnecessary because the solution seeks valley-filling due to the lower prices in evening hours. This evening-hour focus does not imply that we "prohibit" day-time charging, a function that may be desirable to PEVs with long trips. Daytime charging will be treated as conventional (uncontrollable) grid load.

The dynamic programming technique is used to solve this optimization problem, and Fig. 9 shows the optimal controls for the nominal case. The four arrows marked at Hour 26 illustrate that the constraint for the balance of supply and demand, (10), has been satisfied. The difference between the total generation $\left(u_{1}+u_{2}\right)$ and the nominal load is used for PEV charging.

Several properties in the optimal solution are observed:

1) A noticeable amount of PEV charging is saved for the last hour, so that there are enough PEVs available as reserve, which helps to reduce the cost associated with conventional reserve scheduling and dispatch.

2) The scheduling of the electricity generation, $u_{1}$, generally takes advantages of the staircase kinks in the energy price by using as much low-price generation as possible. This phenomenon can be seen in Fig. 9 where the generations below $\$ 28.0 / \mathrm{MWh}$ were fully used all the time. This is true except for the last hour, due to the wind power reserve factor stated above.

3) Due to the fact there is no penalty on early or late PEV charging as long as it happens during the designated horizon, this optimization problem may have multiple solutions. For, example, exchanging $u_{1}(30)$ or $u_{1}(31)$ with $u_{1}(t<30)$ will not alter the cost optimality.

The optimal control, $u_{1}^{*}$, will replace the tracking reference, $P_{\mathrm{L}, \text { nominal }}$, in (7), and this new tracking reference is denoted as $P_{\text {energy, } s}$, in Fig. 7. Furthermore, both $u_{1}^{*}$ and $u_{2}^{*}$ will affect the feed forward component in the middle-level controller for PEV charging.

\section{B. Middle-Level Controller: Load Following}

The middle-level controller plans the charging of the whole PEV fleet so that the sum of the PEV load and grid load

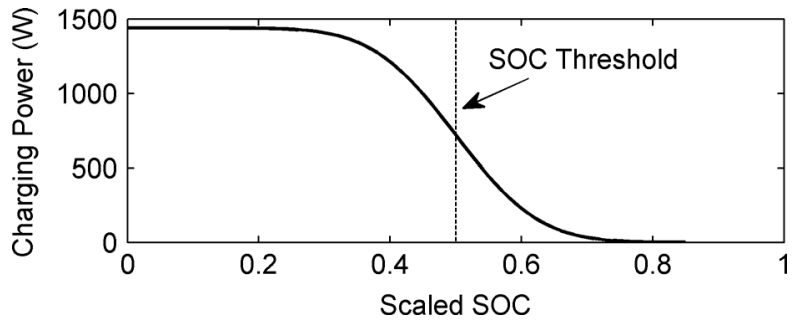

Fig. 10. Charging power allocation rule. This decreasing function is implemented on each smart charger locally, which maps low SOC to a high charging power and vice versa.

matches the generation scheduled by the top-level controller. The middle-level controller consists of two elements: the centralized broadcast (denoted as $C$ in Fig. 7) and the charging power allocation rule ( $G$ in Fig. 7). The former is done by the grid operator, and the latter is implemented by individual smart chargers. This decentralized arrangement allows this scheme to be applied to an indefinite number of PEVs.

1) Centralized Broadcast: This element is the feed forward component for PEV charging, in which an SOC threshold, $S O C_{\mathrm{thr}}$, is derived [see (20)-(21)], and the grid operator broadcasts it to smart chargers to coordinate the charging over the whole PEV fleet:

$$
\begin{aligned}
r & =P_{\mathrm{PEV}}=u_{1}^{*}+u_{2}^{*}-P_{\mathrm{L}, \text { nominal }} \\
C & =\left\{G^{-1}: r \rightarrow S O C_{\mathrm{thr}}\right\} .
\end{aligned}
$$

Equation (20) is an updated version of (10) and states the reference of the PEV charging, $r$, which is essentially the scheduled PEV load. Equation (21) converts $r$ to $S O C_{\mathrm{thr}}$ by inverting the downstream of the system, i.e., the charging power allocation rule, $G$ (detailed in the next section). More specifically, one way to compute $C$ numerically is to search through all possible values of $S O C_{\mathrm{thr}}$ to find out the accumulated PEV load at each time step, and the particular $S O C_{\mathrm{thr}}$ that produces the accumulated PEV exactly the same as $r$ will be the control input chosen to be fed to $G$.

Equations (20) and (21) are computed offline since all needed information can be acquired a priori. It should be pointed out that the grid operator does not need information from every PEV to compute (20)-(21). Only the total number of PEVs, battery capacity, and the three distributions shown in Fig. 1 are required. Furthermore, these parameter/distributions need not be very accurate because the feedback actions can accommodate for the uncertainties.

2) Charging Power Allocation Rule: The idea of the allocation is based on observations from the optimal PEV charging in [40]: allot higher power to vehicles with low SOC and early plug-off time. The low/high SOC level is relative to $S O C_{\mathrm{thr}}$ broadcasted by the grid operator, and the earliness of plug-off time is relative to the end of time horizon in the scheduling optimization, i.e., Hour 32.

The charging power is determined by the hyperbolic tangent curve shown in Fig. 10, which maps low SOC to high charging power and vice versa. In addition, this curve is symmetric to $S O C_{\mathrm{thr}}$ and will shift to the right if $S O C_{\mathrm{thr}}$ rises. $S O C_{\mathrm{thr}}$ serves as the tuning knob to the grid operator for commanding the charging of all PEVs. In addition, despite of the fact all PEVs 
are assumed to have the same battery capacity in this paper, the hyperbolic tangent curve can handle the scenario of PEVs having different battery capacities. The curve has sharp slope around $S O C_{\mathrm{thr}}$, and if a PEV with a large battery is inadequately charged, its charging power will quickly ramp up. Furthermore, the scaling factor, $S F$, defined in (22) allows PEVs that unplug early to charge at a higher power. For example, a vehicle with the plug-off time at Hour 30.5 (6:30 AM) will have $S F$ equals 1.2, i.e., its charging power will be scaled up by $20 \%$. Since it is assumed that the grid operator is capable of deriving the SOC distribution, the amount of additional PEV load due to early plug-off can also be computed and incorporated into the computation for the Centralized Broadcast (i.e., derive $S O C_{\mathrm{thr}}$ ):

$$
S F=\max \left\{1, \frac{(\text { Hour } 32)-(\text { Hour } 23)}{(\text { Plug-Off Time })-(\text { Hour } 23)}\right\} .
$$

To further elaborate the power alslocation rule, the curve in Fig. 10 does not possess optimality under any criterion. In fact, any decreasing curve may do the job, although a smooth curve is preferable for designing the feedback gains in Section III-C. Similarly, the scaling factor can have other forms, for example, a squared term can further favor vehicles with early plug-off times; nevertheless, the current choice in (22) was found sufficient.

\section{Bottom-Level Controller: Grid Frequency Regulation}

The bottom-level controller, denoted as $k_{\mathrm{soc}}$ and $k_{\mathrm{I}, \mathrm{soc}}$ in Fig. 7, is a feedback control, which is designed to mimic the feedback PI-controller in the original grid in Fig. 6. This controller uses the grid frequency deviation as the feedback cue to alter the centralized broadcast signal, $S O C_{\mathrm{thr}}$, so that the PEV charging will temporally speed up or slow down. Furthermore, this controller will be implemented on individual PEVs locally, meaning that the feedback control will continue to regulate the grid frequency even if the (centralized) reference signal is delayed or interrupted.

The challenge of designing the feedback gains, $k_{\mathrm{soc}}$ and $k_{\mathrm{I}, \mathrm{soc}}$, lies in the fact that the chosen curve in Fig. 10 makes the input/output relation of $G$ nonlinear; so, $k_{\mathrm{soc}}$ and $k_{\mathrm{I} \text {,soc }}$ must be designed for robust performance under varying plant sensitivity. Indeed, $k_{\mathrm{soc}}$ and $k_{\mathrm{I} \text {, soc }}$ are designed based on the sensitivity analysis.

1) Feedback Gains for PEV Charging: The PI-controller gains, $k$ and $k_{\mathrm{I}}$, in Fig. 6 provide the inspiration to derive $k_{\mathrm{soc}}$ and $k_{\mathrm{I}, \mathrm{soc}}$ for PEV charging.

The PI-controller gains, $k$ and $k_{\mathrm{I}}$, in Fig. 6 can be found by existing approaches, such as pole placement or root locus technique; furthermore, the proportional gain, $k$, has the physical meaning as the inverse of frequency sensitivity to the regulation power, shown in (23):

$$
k=\frac{\partial P_{\text {reserve }}}{\partial \omega} .
$$

In the proposed concept of controlling PEV charging to regulate the grid frequency, the proportional gain $k_{\text {soc }}$ needs to embody the physical meaning as the inverse of frequency

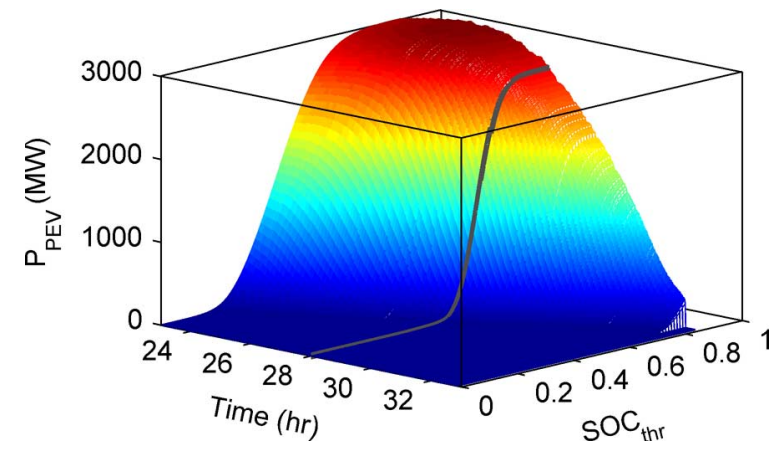

Fig. 11. All possible values of $P_{\mathrm{PEV}}$ in the time window of generation scheduling.

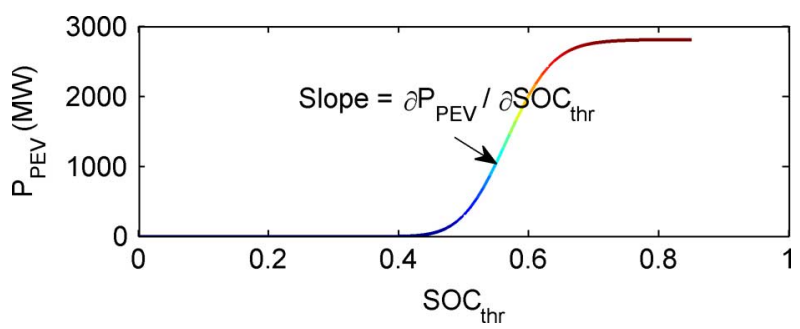

Fig. 12. Sensitivity of $P_{\mathrm{PEV}}$ to $S O C_{\mathrm{thr}}, \mathbb{S}$, at Hour 28 .

sensitivity to the SOC threshold broadcast, which can be done through dividing $k$ by the sensitivity of $P_{\mathrm{PEV}}$ to $S O C_{\mathrm{thr}}, S$, as shown in (24) and (25), where $S$ can be numerically computed (illustrated in the next section). The integral gain, $k_{\mathrm{I}, \mathrm{soc}}$, can be found in the same way, as shown in (26):

$$
\begin{aligned}
\mathbb{S} & =\frac{\partial P_{P E V}}{\partial S O C_{\mathrm{thr}}} \\
k_{\mathrm{soc}} & =\frac{\partial S O C_{\mathrm{thr}}}{\partial \omega}=k \frac{\partial S O C_{\mathrm{thr}}}{\partial P_{\mathrm{PEV}}}=\frac{k}{\mathbb{S}} \\
k_{\mathrm{I}, \mathrm{soc}} & =\frac{k_{1}}{\mathbb{S}} .
\end{aligned}
$$

In fact, $\mathbb{S}$ is the linearization of $G$, and (25) and (26) ensure that both systems in Figs. 6 and 7 have the same closed-loop poles. Furthermore, due to the much faster response in PEV charging than conventional reserves, it is possible to achieve better performance in frequency regulation by choosing faster closed-loop poles when designing $k_{\mathrm{soc}}$ and $k_{\mathrm{I} \text {,soc }}$.

2) Sensitivity of PEV Load: Figs. 11 and 12 explain how to compute $\mathbb{S}$ numerically. Fig. 11 is obtained by offline computation, which uses the distributions of the plug-in time, plug-off time, and $S O C_{\mathrm{ini}}$ derived in Section II-A to find all possible $P_{\mathrm{PEV}}$ values by searching through every $S O C_{\mathrm{thr}}$ level in Hours 23-32. Fig. 12 is then extracted from Fig. 11 at a specific time (Hour 28), and the slope of the extracted curve is $\mathbb{S}$ to be used in (25) and (26).

Due to the fact that $\mathbb{S}$ changes over time, the feedback gains $k_{\mathrm{soc}}$ and $k_{\mathrm{I}, \mathrm{soc}}$ are not constant. A simplified constant gain controller is further derived by using the largest value of $\mathbb{S}$ in (25) and (26), which is easier to implement. It has been confirmed in the previous study [19] that, on the Nyquist plot, the simplified constant-gain design still possesses a large stability radius for the system in Fig. 7. 
TABLE I

Simulation CASES

\begin{tabular}{cccc}
\hline \hline Case & $\begin{array}{c}\text { PEV } \\
\text { Penetration }\end{array}$ & $\begin{array}{c}\text { Wind Power } \\
\text { Penetration }\end{array}$ & Control Implementation \\
\hline A (Ref.) & $25 \%$ & None & No control integration \\
B & $25 \%$ & $10 \%$ & Only first two levels of control \\
C & $25 \%$ & $10 \%$ & All three levels of control \\
\hline \hline
\end{tabular}

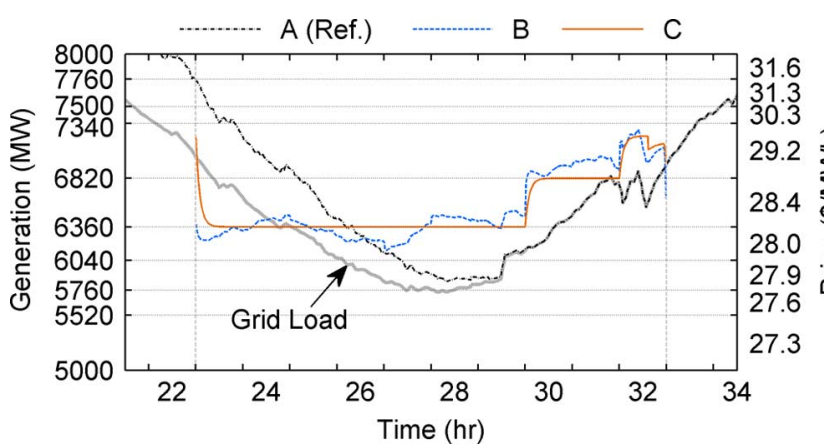

Fig. 13. Generation dispatch from conventional sources. The oscillations in Cases A and B are due to fluctuations in the grid load and wind power. Case $\mathrm{C}$ throttles the PEV charging so that the generation can closely follow the optimal scheduling in Fig. 9.

\section{Simulations}

Three cases, with different penetration levels of PEV and wind power and different degrees of control integration, are simulated to demonstrate the effectiveness of the three-level controller. These three cases are summarized in Table I. Case A serves as the reference with unmitigated PEV charging. Cases B and $\mathrm{C}$ both have $25 \% \mathrm{PEV}$ s in the transportation sector and $10 \%$ wind power in the electricity generation mix; however, Case B has only the top and middle-level controller implemented but not the bottom level, and the conventional reserve is still needed for frequency regulation, whereas Case $\mathrm{C}$ has all three levels of controllers and no conventional reserve. ${ }^{1}$

All three cases have the same percentage of PEVs fully charged, $99.5 \%$, but their performances in generation dispatch, frequency regulation, and costs are different.

Fig. 13 shows the actual dispatch, including both the electricity generation and reserve from conventional sources. Case A has substantial generation increase before Hours 23 because PEVs start charging in early evenings, while Cases $B$ and $C$ have the PEV load properly confined within Hours $23-32$. However, Case B does not achieve the maximum benefit because fluctuations in the grid load and wind power demand dispatching conventional reserves. Case $\mathrm{C}$ has feedbacks to throttle the PEV charging and the generation closely follows the optimal scheduling $\left(u_{1}^{*}\right)$, except the beginning of Hours 23,30 and 32 when $u_{1}^{*}$ has large changes and the slow time constant $\tau_{1}$ limits the ramping of the electricity generation.

Fig. 14 highlights the frequency regulation results. Cases $\mathrm{A}$ and $\mathrm{B}$ both use the conventional reserve and have similar

\footnotetext{
${ }^{1}$ In Case C, the conventional reserve is switched off except the latter half hour in Hour 32, when most PEVs are fully charged and unavailable for providing grid frequency regulation. The simulation detects the frequency deviation being larger than $1 \mathrm{~Hz}$ (see Fig. 14), and it then switches the conventional reserve back on.
}

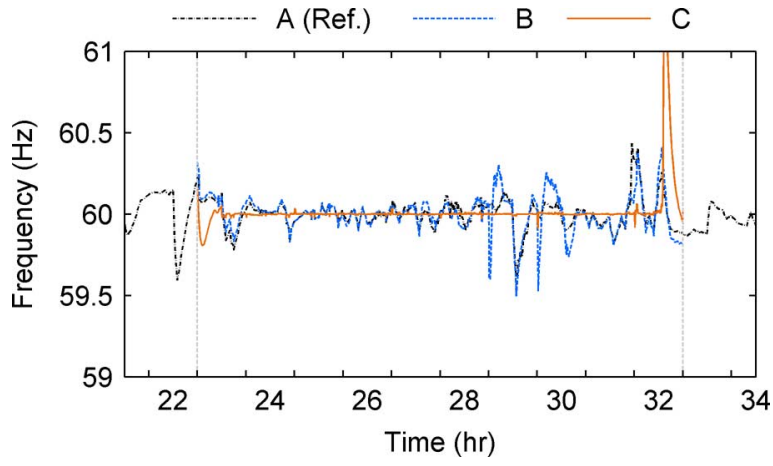

Fig. 14. Grid frequency regulation. Case C outperforms Cases A and B most of the time because controlling PEV charging has faster response than dispatching conventional reserves.

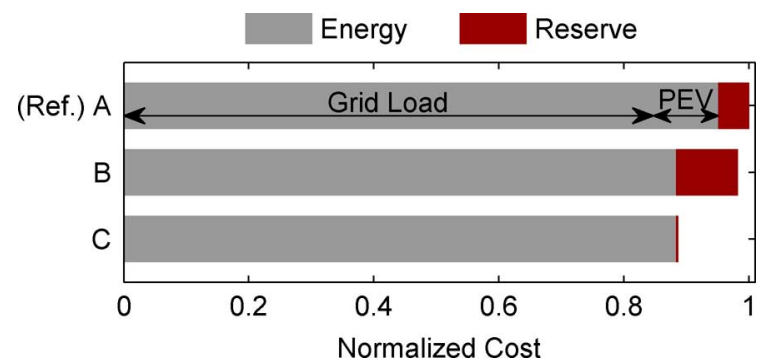

Fig. 15. Total electricity generation costs of various degrees of control integration.

frequency deviations, whereas the grid frequency in Case $\mathrm{C}$ is regulated by controlling the PEV charging and has much smaller deviation most of the time. However, the performance in Case $\mathrm{C}$ deteriorates at both ends of the valley hour; Hour 23 is due to the singularity of $G$ at that particular time (the input/output relation is close to zero), and Hour 32 is because most PEVs are fully charged.

Fig. 15 shows total generation costs of the three simulation cases. Each case two cost components, one for the energy and the other for the reserve. Case A has energy cost for serving the grid load and the uncontrolled PEV charging and reserve cost for covering fluctuations in the grid load. Case B, due to the utilization of wind power, has smaller energy cost but larger reserve cost. In fact, the cost reduction in energy is almost cancelled by the increase in reserve. Case $\mathrm{C}$ has energy cost similar to Case $\mathrm{B}$ because of using wind power and has a very low reserve cost because the controlled PEV charging eliminates the use of conventional reserves for most of the time. Notice that the cost in Case $\mathrm{C}$ is based on the assumption that it is free to control PEV charging to support reserves and no rewards are given to PEV owners. Hence, Case $\mathrm{C}$ can be interpreted as a lower bound for the system operation costs with PEVs. If compensation is paid to PEV owners for providing reserves, the compensation should not be more than the cost difference between Case B and Case $\mathrm{C}$, else it will be more economical to use conventional generators as reserves.

To further illustrate the value of the synergy between PEV and wind power, Fig. 16 shows the cost difference between Case $\mathrm{C}$ and $\mathrm{B}$ at various penetration levels of PEV and wind power with the proposed hierarchical controller implemented. Several markers of PEV and renewable energy targets [15], [41], [42] are also shown in the figure. The cost difference is normalized 


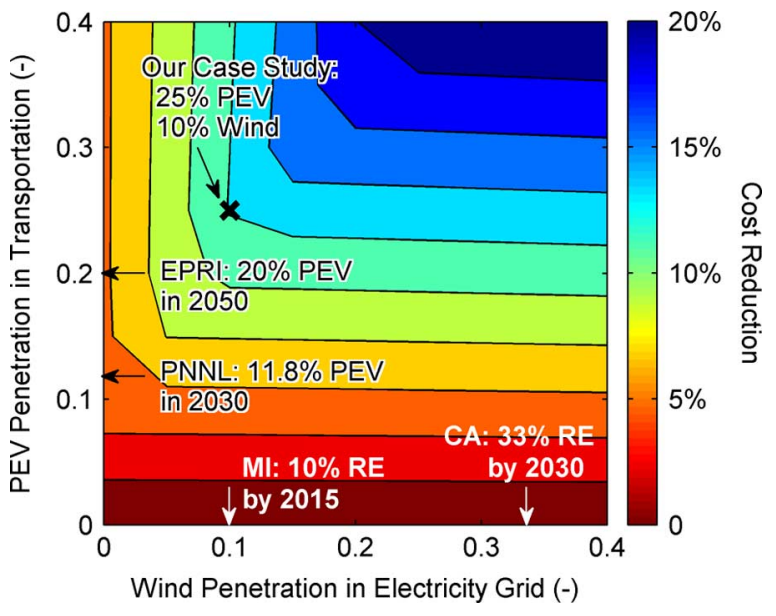

Fig. 16. Cost difference between Case C and B (normalized). Significant cost reduction is achieved when the PEV fleet and wind power grow together.

to the cost of Case B. This figure shows that the cost reduction merely exists when only one of the two green technologies is present on the grid, but the cost can reach a remarkable $20 \%$ reduction when both technologies are at high penetrations and the synergy fully utilized.

\section{CONCLUSION}

This paper proposes a three-level controller to realize the synergy between the controllable PEV charging and the intermittent renewable wind energy: the top-level controller minimizes the generation cost and finds scheduling of (non-renewable) generation and wind power; the middle-level controller allots charging power to individual PEVs based on their battery SOC and plug-off time to achieve load following; and, the bottom-level controller uses feedbacks to control PEV charging in real time to regulate the grid frequency. The effectiveness of this controller is validated by simulations on a state-wide grid mode based on realistic data in Michigan. Major results are summarized as follows:

1) The proposed control algorithm is implementable.

The algorithm comprehensively considers major grid dynamics at various time scales, including the hourly scheduling, sub-hour PEV charging scheduling, and real-time grid frequency regulation, and it is designed to have enough instruments to handle the real world uncertainties. Furthermore, the algorithm consists of an explicit PEV charging rule (the hyperbolic tangent curve) that is simple enough to be programmed on smart chargers.

2) Most PEVs are fully charged.

99.5\% of PEVs are fully charged even though the PEV fleet is used to regulate grid frequency, meaning that the idle generation capacity in evenings can accommodate quite many PEVs if the charging is well controlled.

3) Grid frequency regulation is improved.

The response of controlling PEV charging is much faster than dispatching conventional reserves, which enables the much improved frequency regulation.

4) The synergy achieves substantial cost reduction.

To the authors' best knowledge, the proposed control algorithm is one of the earliest in the literature that realizes the synergy of PEV and wind power. Furthermore, the significant cost reduction in Figs. 15 and 16 indicates that the PEV fleet and renewable power should grow together to realize their full benefits.

This paper demonstrated the value of fully explore the synergy between PEV and wind power. The control concept is not limited to wind power and can be extended to other intermittent renewable sources. Some open questions remain to be answered. A key requirement is to make the controller adaptive to handle the non-zero mean forecast error in wind power, which may hurt the PEV charging completion in the situation of wind under-production.

\section{REFERENCES}

[1] W. Kempton and J. Tomic, "Vehicle-to-Grid power fundamentals: Calculating capacity and net revenue," J. Power Sources, vol. 144, pp. 268-279, 2005.

[2] W. Kempton and J. Tomic, "Vehicle-to-Grid power implementation: From stabilizing the grid to supporting large-scale renewable energy," J. Power Sources, vol. 144, pp. 280-294, 2005.

[3] S. Hadley and A. Tsvetkova, "Potential impacts of plug-in hybrid electric vehicles on regional power generation," Electr. J., vol. 22, pp. 56-68, 2009.

[4] M. Kintner-Meyer, K. Schneider, and R. Pratt, Impacts Assessment of Plug-In Hybrid Vehicles on Electric Utilities and Regional U.S. Power Grids Part 1: Technical Analysis, Pacific Northwest National Laboratory, 2007.

[5] T. Ibaraki and N. Katoh, Resource Allocation Problems: Algorithmic Approaches. Cambridge, MA: MIT Press, 1988.

[6] W. Burke and D. Auslander, "Residential electricity auction with uniform pricing and cost constraints," in Proc. North American Power Symp., Starkville, MS, 2009, pp. 1-6.

[7] R. Maheswaran and T. Basar, "Decentralized network resource allocation as a repeated noncooperative market game," in Proc. 40th IEEE Conf. Decision and Control, Orlando, FL, 2001, pp. 4565-4570.

[8] M. Pinedo, Scheduling: Theory, Algorithms, and Systems, 3rd ed. New York: Springer, 2008.

[9] I. Vermeulen, S. Bohte, S. Elkhuizen, H. Lameris, P. Bakker, and J. Poutré, "Adaptive resource allocation for efficient patient scheduling," Artif. Intell. Med., vol. 46, pp. 67-80, 2009.

[10] M. Carter and S. Lapierre, "Scheduling emergency room physicians," Health Care Manage. Sci., vol. 4, pp. 347-360, 2001.

[11] M. Rossetti, G. Trzcinski, and S. Syverud, "Emergency department simulation and determination of optimal attending physician staffing schedules," in Proc. 31st Winter Simulation Conf., Phoenix, AZ, 1999 , pp. 1532-1540.

[12] D. Callaway and I. Hiskens, "Achieving controllability of electric loads," Proc. IEEE, vol. 99, pp. 184-199, 2011.

[13] The Detroit Edison Company, Rate Book for Electric Service, Report, 2011.

[14] Southern California Edison. Rate Information-Residential Rates Electric Vehicles, 2011. [Online]. Available: http://www.sce.com/CustomerService/rates/residential/electric-vehicles.htm.

[15] Environmental Assessment of Plug-In Hybrid Electric Vehicles, Volume 1: Nationwide Greenhouse Gas Emissions, Electric Power Research Institute (EPRI), 2007, \#1015325.

[16] D. Lemoine, D. Kammen, and A. Farrell, "An innovation and policy agenda for commercially competitive plug-in hybrid electric vehicles," Environ. Res. Lett., vol. 3, pp. 14003-14013, 2008.

[17] M. Galus and G. Andersson, "Demand management of grid connected plug-in hybrid electric vehicles (PHEV)," in Proc. IEEE Energy 2030 Conf., Atlanta, GA, 2008, pp. 1-8.

[18] S. Han, S. Han, and K. Sezaki, "Development of an optimal vehicle-toGrid aggregator for frequency regulation," IEEE Trans. Smart Grid, vol. 1, pp. 65-72, 2010.

[19] C.-T Li, C. Ahn, H. Peng, and J. Sun, "Decentralized charging of plug-in electric vehicles," in Proc. Dynamic System and Control Conf., Arlington, VA, 2011.

[20] W. Katzenstein and J. Apt, The Cost of Wind Power Variability, Carnegie Mellon Electricity Industry Center, 2010, \#CEIC-10-05.

[21] R. Doherty and M. O'Malley, "A new approach to quantify reserve demand in systems with significant installed wind capacity," IEEE Trans. Power Syst., vol. 20, pp. 587-595, 2005. 
[22] R. Wiser and M. Bolinger, 2009 Wind Technologies Market Report, Department of Energy, 2010, \#DOE/GO-102010-3107.

[23] G. Giebel, R. Brownsword, G. Kariniotakis, M. Denhard, and C. Draxl, The State-of-the-Art in Short-Term Prediction of Wind Power-A Literature Overview, Risø National Laboratory, Report, 2011, 2nd edition.

[24] J. Hetzer, D. Yu, and K. Bhattarai, "An economic dispatch model incorporating wind power," IEEE Trans. Energy Convers., vol. 23, pp. 603-611, 2008.

[25] F. Bouffard and F. Galiana, "Stochastic security for operations planning with significant wind power generation," IEEE Trans. Power Syst., vol. 23, pp. 306-316, 2008.

[26] J. Matevosyan and L. Söder, "Minimization of imbalance cost trading wind power on the short-term power market," IEEE Trans. Power Syst., vol. 21, pp. 1396-1404, 2006.

[27] P. Pinson, C. Chevallier, and G. Kariniotakis, "Trading wind generation from short-term probabilistic forecasts of wind power," IEEE Trans. Power Syst., vol. 22, pp. 1148-1156, 2007.

[28] E. Bitar, A. Giani, R. Rajagopal, D. Varagnolo, P. Khargonekar, K. Poolla, and P. Varaiya, "Optimal contracts for wind power producers in electricity markets," in Proc. 49th IEEE Conf. Decision and Control, Atlanta, GA, 2010, pp. 1919-1926.

[29] V. Miranda and P. Hang, "Economic dispatch model with fuzzy wind constraints and attitudes of dispatchers," IEEE Trans. Power Syst., vol. 20, pp. 2143-2145, 2005.

[30] C.-T. Li, C. Ahn, H. Peng, and J. Sun, "Integration of plug-in electric vehicle charging and wind energy scheduling on electricity grid," in Proc. IEEE PES Conf. Innovative Smart Grid Technologies (ISGT 2012), Arlington, VA, 2012.

[31] T.-K. Lee, Z. Baraket, T. Gordon, and Z. Filipi, "Stochastic modeling for studies of real-world PHEV usage: driving schedules and daily temporal distributions," IEEE Trans. Veh. Technol., accepted for publication.

[32] GM-Volt Website, Latest Chevy Volt Battery Pack and Generator Details and Clarifications, 2007. [Online]. Available: http://gm-volt. com/2007/08/29/latest-chevy-volt-battery-pack-and-generator-details-and-clarifications/.

[33] SAE Electric Vehicle Conductive Charge Coupler, SAE Standard J1772, 2001.

[34] National Renewable Energy Laboratory (NREL), Eastern Wind Dataset, 2010. [Online]. Available: http://www.nrel.gov/wind/integrationdatasets/eastern/methodology.html.
[35] H. Daneshi and A. Daneshi, "Real time load forecast in power system," in Proc. 3rd Int. Conf. Electric Utility Deregulation and Restructuring and Power Technologies, Nanjing, China, 2008, pp. 689-695.

[36] S. Hadley, The Oak Ridge Competitive Electricity Dispatch (ORCED) Model, Oak Ridge National Laboratory, 2008, \#ORNL/TM-2007/230.

[37] Potomac Economics, 2010 State of the Market Report for the MISO Electricity Markets, 2011

[38] P. Kundur, N. J. Balu, and M. G. Lauby, Power System Stability and Control. New York: McGraw-Hill, 1994.

[39] J. Short, D. Infield, and L. Freris, "Stabilization of grid frequency through dynamic demand control," IEEE Trans. Power Syst., vol. 22, pp. 1284-1293, 2007.

[40] C. Ahn, C.-T Li, and H. Peng, "Optimal decentralized charging control algorithm for electrified vehicles connected to smart grid," J. Power Sources, vol. 196, pp. 10369-10379, 2011.

[41] T. Becker, I. Sidhu, and B. Tenderich, Electric Vehicles in the United States a New Model with Forecasts to 2030, Center for Entrepreneurship \& Technology, University of California, Berkeley, 2009.

[42] Database of State Incentives for Renewables \& Efficiency, 2010. [Online]. Available: http://www.dsireusa.org.

Chiao-Ting Li (M'12) is pursuing the Ph.D. degree in the Department of Mechanical Engineering, University of Michigan, Ann Arbor.

Changsun Ahn is a Postdoctoral Researcher in the Department of Mechanical Engineering, University of Michigan, Ann Arbor

Huei Peng is a Professor in the Department of Mechanical Engineering, University of Michigan, Ann Arbor.

Jing Sun (F'04) is a Professor in the Department of Naval Architecture and Marine Engineering, University of Michigan, Ann Arbor. 